\title{
土壤铁矿物形态转化影响有机碳固定研究进展
}

\author{
宋旭昕 ${ }^{1,2,3}$, 刘同旭 ${ }^{1, *}$ \\ 1 广东省科学院生态环境与土壤研究所,华南土壤污染控制与修复国家地方联合工程研究中心,广东省农业环境综合治理重点实验室, 广州 \\ 510650 \\ 2 桂林理工大学, 旅游与风景园林学院, 桂林 541004 \\ 3 桂林理工大学, 植物与生态工程学院, 桂林 541004
}

\begin{abstract}
摘要: 铁是地壳中丰度第四高的元素, 其可通过多种方式影响土壤有机碳累积,尤其铁氧化物与土壤有机碳相互作用形成的稳 定有机-矿物复合物, 被认为是土壤可溶性有机碳长期固定的关键地球化学机制。促进土壤固定有机碳不仅可以提高土壤质量 和肥力, 还是应对全球气候变化的主要策略之一。然而,铁活跃的氧化还原反应和多样化的赋存形态,使其转化过程对土壤有 机碳累积和稳定性的影响结果受到诸多生物和非生物因素调控。从不同角度,结合多学科的研究成果,综述了近年来国内外关 于铁矿物形态转化影响土壤有机碳固定的相关研究,包括铁矿物形态转化过程、土壤有机碳固定机制、铁矿物形态转化影响土 壤有机碳固定的机制及其主要影响因素(各种环境条件、自身的铁矿物性质、碳源质量等方面),强调铁在土壤有机碳固定过程 中的重要作用。对铁固定土壤有机碳的相关研究提出了建议,为今后研究提供相关参考。
\end{abstract}

关键词 : 铁矿物; 铁氧化还原;形态转化;有机碳固定

\section{Effects of soil iron mineral transformation on organic carbon sequestration: a review}

\author{
SONG Xuxin ${ }^{1,2,3}$, LIU Tongxu ${ }^{1, *}$ \\ 1 National-Regional Joint Engineering Research Center for Soil Pollution Control and Remediation in South China, Guangdong Key Laboratory of Integrated \\ Agro-environmental Pollution Control and Management, Institute of Eco-environmental and Soil Sciences, Guangdong Academy of Sciences, Guangzhou \\ 510650 , China \\ 2 College of Tourism and Landscape Architecture, Guilin University of Technology, Guilin 541004, China \\ 3 College of Plant and Ecological Engineering, Guilin University of Technology, Guilin 541004, China
}

\begin{abstract}
Iron is the fourth most abundant element in the earth's crust, which can affect soil organic carbon accumulation in a variety of ways. The stable organic-mineral complex formed by the interaction between iron oxides and soil organic carbon is considered to be the key mechanism for long-term storage of soil dissolved organic carbon. Promoting soil organic carbon sequestration can not only improve soil quality and fertility, but also be an important strategy to address global climate change. However, the effects of iron mineral transformation on the accumulation and stability of soil organic carbon are regulated by many biotic and abiotic factors, due to the active redox reactions of iron and its diverse forms. This paper summarized the recent progresses on the effects of iron mineral transformation on soil organic carbon sequestration, from different perspective and multi-disciplinary research results, including the transformation processes of iron minerals, the mechanisms of organic carbon sequestration, and the main regulative factors of iron mineral transformation affecting soil
\end{abstract}

基金项目: 国家自然科学基金(32001141); 中国博士后科学基金(2020M682636); 广东省科学院实施创新驱动发展能力建设专项(2020GDASYL20200103081)

收稿日期:2019-11-12; 采用日期:2021-05-20

* 通讯作者 Corresponding author.E-mail: txliu@ soil.gd.cn 
organic carbon sequestration ( environmental conditions, iron mineral properties, carbon substrate quality, etc.), and emphasized the important roles of iron in the soil organic carbon sequestration. Finally, this paper puts forward some suggestions for the study of soil organic carbon sequestration by iron for future research.

Key Words : iron mineral ; iron redox ; transformation ; organic carbon sequestration

铁是地壳中含量第四的元素,其氧化还原性质活跃 ${ }^{[1]}$ 。以往研究发现其与物质转化、污染物降解、重金 属的吸附与解吸附等密切相关而受到重视 ${ }^{[2-3]}$ 。近年来随着全球变暖, $\mathrm{CO}_{2}$ 升高, 土壤碳固定成为生态学研究 热点,逐渐有研究关注活跃的金属元素铁对土壤有机碳固定的调节作用。铁矿物被称为有效的“锈汇”,能捕 获陆地来源的有机碳, 是长期土壤有机碳储存的关键因素 ${ }^{[4-5]}$, 可作为主要的预测因子 ${ }^{[6]}$, 尤其是在许多中性 和酸性土壤。铁可以通过多种方式调节土壤有机碳固定,其中铁矿物与有机物相互作用形成的铁矿物-土壤 有机碳复合体会阻碍微生物利用土壤有机碳, 导致更高的土壤碳储量, 被认为是长期碳固定的主要机制 ${ }^{[7-9]}$ 。 本文将对铁矿物形态转化影响土壤有机碳固定的机制及影响因素进行阐述,阐明铁对土壤有机碳固定的重要 性, 明确当前相关研究的进展和热点问题,并根据已有研究提出相应的建议。

\section{1 铁矿物的形态转化过程}

铁循环包括 $\mathrm{Fe}$ (III) 还原和 $\mathrm{Fe}$ (II) 氧化两大过程, 其本质是质子、电子等信号物质在分子间的传递, 从而 驱动碳氮转化 ${ }^{[10]}$ 。通常在高氧条件下, $\mathrm{Fe}$ ( II) 会快速化学氧化成 $\mathrm{Fe}(\mathrm{III})$, 而在缺氧或有氧化剂存在的条件 下 $\mathrm{Fe}$ (II) 氧化主要通过生物和非生物的共同作用 ${ }^{[11-13]}$ 。在中性 $\mathrm{pH}$ 的条件下催化 $\mathrm{Fe}$ ( II) 氧化的微生物类型 主要有低氧逸度下的微氧亚铁氧化菌、光条件下的光合型亚铁氧化菌和厌氧条件下的硝酸盐还原亚铁氧化 菌 ${ }^{[11,14]}$ 。在厌氧条件下硝酸盐还原亚铁氧化菌不受光环境限制, 因此比光养菌更丰富 ${ }^{[15]}$ 。硝酸盐依赖型亚 铁氧化菌会驱动 $\mathrm{Fe}(\mathrm{II})$ 氧化耦合 $\mathrm{NO}_{3}^{-}$还原过程, 同时 $\mathrm{NO}_{3}^{-}$还原产物 $\mathrm{NO}_{2}^{-}$也会与 $\mathrm{Fe}(\mathrm{II})$ 发生直接的化学反 应,生成次生铁矿、 $\mathrm{NO}$ 和 $\mathrm{N}_{2} \mathrm{O}$ 等 ${ }^{[16]}$ 。 $\mathrm{Liu}^{[17]}$ 等定量评估厌氧条件下硝酸盐还原亚铁氧化过程中化学反硝化 作用和微生物反应的相对贡献, 结果表明 $\mathrm{NO}_{2}^{-}$化学过程对 $\mathrm{Fe}$ ( II) 氧化的相对贡献大于生物过程。 $\mathrm{Fe}(\mathrm{III})$ 还 原过程也是包括化学驱动和生物驱动两个机制, 在高氧条件下, $\mathrm{Fe}(\mathrm{III})$ 还原只在有还原剂或有机酸存在时通 过光化学作用发生 ${ }^{[18]}$, 而在近中性 $(\mathrm{pH}>4.0)$ 的厌氧条件下, $\mathrm{Fe}$ ( III) 还原主要通过异化铁还原菌 (如 Shewanella/Geobacter species) 或地球化学还原物驱动, 如腐殖酸和硫化物 ${ }^{[19-21]}$ 。目前的研究中也证实了铁还 原菌种确实存在铁还原基因 $m t r A 、 m t r B$ 和 $c y m A$ 等。这些铁还原菌在还原 $\mathrm{Fe}$ ( III) 的同时会大量利用有机碳 源, 有些细菌甚至可以利用无机碳源作为电子供体, 比如 $\mathrm{H}_{2} 、 \mathrm{NH}_{4}^{+}$等。铁还原菌在环境中的普遍存在以及铁 在地壳中含量丰富,致使其对铁元素的生物地球化学循环尤其重要。

自然状态下土壤中的铁会快速氧化而以氧化铁的形态存在, 常见的氧化铁包括短程有序 (如水铁矿) 和 长程有序 (如纤铁矿、针铁矿、赤铁矿等 ${ }^{[22-23]}$ 。土壤中氧化铁的形态包括无定型态氧化铁 $\left(\mathrm{Fe}_{0}\right)$ 、游离态氧化 铁 $\left(\mathrm{Fe}_{\mathrm{d}}\right)$ 和络合态氧化铁 $\left(\mathrm{Fe}_{\mathrm{p}}\right)$ 。无定型氧化铁是指能用草酸铵提取的氧化铁, 其活性较高, 比表面积较大; 游离氧化铁是指能用连二亚硫酸钠-柠檬酸钠-重碳酸钠 (DCB) 提取的氧化铁, 是土壤中排除在层状硅酸盐

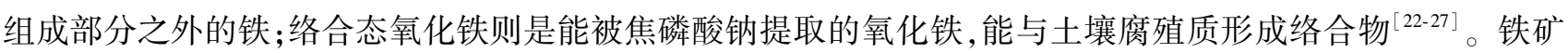
物的产生不仅是土壤 $\mathrm{pH}$ 、氧化还原电位和根系分泌物对原生矿物的风化作用,也是土壤微生物介导的生物化 学过程 ${ }^{[28-30]}$ 。

\section{2 土壤有机碳固定的基本原理}

土壤有机碳( Soil organic carbon; SOC) 因其较慢的周转时间而成为陆地最大的碳库, 分别是大气碳库和 生物碳库的 3.2 倍和 4.4 倍, 其较小改变就会显著影响大气二氧化碳浓度 ${ }^{[31-34]}$ 。同时土壤有机碳含量被认为 
是土壤质量的重要指标 ${ }^{[35]}$, 因此将大气中二氧化碳固定到土壤中可能是促进土壤肥力和缓解全球气候变化 的一个有效措施 ${ }^{[36]}$ 。早期研究广泛认为植物残体自身化学组成和分子结构的抵抗性决定土壤有机碳的储存 时间 ${ }^{[37-38]}$, 抵抗性化合物丰富的生态系统也被认为会促进更多大气二氧化碳固定到土壤中 (选择性保留机 制) ${ }^{[39-40]}$ 。然而, 最新研究认为选择性保留机制可能仅在分解初期起主导作用 ${ }^{[41-42]}$, 植物材料即使再抗降解 也终将被分解, 只是时间长短问题, 其稳定性是相对的 ${ }^{[43]}$ 。因此, 阻碍微生物接触有机质以及创造不利于微 生物分解的环境成为有机碳固定的重要策略。本文中将重点关注铁循环参与的碳固定过程, 并将其分为: (1)二氧化碳的同化:大气二氧化碳被转化为土壤有机碳; (2) 矿物质结合可溶性有机碳; 以及 (3) 土壤团聚 体的形成, 以上过程中包括化学反应, 物理保护以及微生物的作用。

\section{1 二氧化碳同化}

二氧化碳同化是将二氧化碳转化为碳水化合物的过程, 改善生态系统的碳储存。碳固定的潜力主要由光 合作用中初级生产者同化的大气二氧化碳量和分解作用中释放回大气的二氧化碳量之间的平衡所决定。除 地上植物光合作用外, 在不同生态系统也广泛存在自养微生物, 二氧化碳是其主要或唯一的碳源, 其可通过多 种途径同化二氧化碳, 合成细胞物质。而微生物自身被认为是稳定土壤有机碳的重要前体。 $\mathrm{Liang}^{[44]}$ 等的研 究表明土壤中大多数持久性碳是通过土壤微生物碳百作用产生的。最新观点认为微生物的死亡残体在土壤 中有更长的周转时间, 可以直接附着在矿物质颗粒表面, 从而产生新的有机一矿物结合体, 促进稳定土壤碳库 的积累 ${ }^{[45-46]}$ 。因此,微生物同化二氧化碳的过程是土壤稳定有机碳的重要形成途径。

\section{2 矿物质与有机碳结合}

经典的地球系统模型认为碳输人质量、气候因素、土壤环境是影响土壤有机碳储量的主要因素 ${ }^{[47-49]}$ 。然 而,最近 Nature Geoscience 上一篇在全球尺度上的试验研究表明地球化学因素在调节土壤有机碳储存起到不 可忽视作用 ${ }^{[50]}$ 。矿物质与有机碳结合是稳定土壤有机碳抵抗降解的重要机制。矿物质和有机质可能通过吸 附作用形成复杂的复合体, 铁氧化物的活性表面位点可以吸附溶解有机质, 是土壤有机碳积累的重要过程。 机制主要包括六种:配位体交换,离子交换, 阳离子架桥,范德华力, 氢键和疏水作用 ${ }^{[43,51-59]}$ 。然而, Wagai 和 Mayer ${ }^{[60]}$ 的研究表明在金属氧化物表面简单的吸附有机质不能完全解释铁氧化物对碳储存的关键作用。在 氧化还原周期性波动的自然环境中, 可溶性有机碳与铁氧化物的相互作用可能不仅包括吸附作用,也包括在 氧化过程中可溶性有机碳与新形成的活性三价铁氧化 (尤其是低结晶铁氧化物) 发生共沉淀, 形成闭蓄态有 机碳从而降低有机碳的生物有效性, 促进有机碳固定 ${ }^{[9,61]}$ 。但当前关于共沉淀的作用知之甚少。与吸附形 成的复合物不同,有机质和铁共沉淀会改变新形成的铁 (氢) 氧化物的粒径和结构顺序 ${ }^{[62-63]}$ 。 $\mathrm{Han}^{[64]}$ 等人发 现共沉淀形成的 Fh-OC 结合物比吸附形成的复合物更能抵抗化学还原。水解共沉淀和高 $\mathrm{C} / \mathrm{Fe}$ 比例下形成 的水铁矿一土壤有机碳复合体结构会更稳定, 有更高的 $\mathrm{C} / \mathrm{Fe}^{[65]}$ 。然而, 也有研究表明吸附和共沉淀对有机质 的影响可能依赖于有机质的组成。

2.3 团聚体形成

土壤团聚体按粒径大小分为大团聚体 $(>250 \mu \mathrm{m})$ 和微团聚体 $(<250 \mu \mathrm{m})$ 。其中 $70 \%$ 以上的土壤有机碳 存在于 $<53 \mu \mathrm{m}$ 的微团聚体中 (即矿物质结合态有机碳, 见 2.2), 但微团聚体会被胶结成大团聚体。铁氧化物 因其表面活性高, 是重要的无机胶结物, 起到絮凝剂的作用,将细颗粒与无机和/或有机分子结合形成团聚 体 ${ }^{[66]}$ 。因此, 通过金属氧化物 (尤其是铁氧化物) 引起的微团聚对有机碳进行物理保护是土壤有机碳累积和 稳定的重要机制之一。团聚体不仅物理保护有机碳以防微生物和酶攻击 ${ }^{[60,67]}$, 也会影响微生物群落结构, 限 制氧气扩散和调节营养循环 ${ }^{[43,68-69]}$ 。土壤团聚体的形成反应了土壤中有机碳稳定是生物、化学和物理机制的 协同作用 ${ }^{[70-71]}$ 。

\section{3 铁矿物形态转化影响土壤有机碳转化的机制}

铁的生物地球化学过程会通过以下三方面作用影响土壤有机碳转化: (1) 在不同氧气条件下铁与碳发生 
的生物-化学反应; (2) 影响矿物质结合态有机质的形成和分解; (3) 影响团聚体的形成和解离。

\section{1 生物-化学反应}

铁循环影响碳转化的过程主要包括: (1) 异化铁还原耦合有机碳矿化 (光化学、有机配体和厌氧微生物异 养分解促进反应);(2) 芬顿反应产生活性氧促进有机碳矿化; (3) 亚铁氧化耦合二氧化碳固定 (光合型微生 物、微氧型微生物和厌氧硝酸盐依赖型微生物驱动反应 $)^{[72-74]}$ 。在不同氧气和光照条件下, 铁和碳可能发生 如下生物-化学反应过程 ${ }^{[75]}$,从而改变碳形态 (表 1)。

表 1 不同氧气条件下铁和碳发生的生物-化学反应

Table 1 The biochemical reactions between iron and carbon under different oxygen conditions

\begin{tabular}{|c|c|c|c|c|}
\hline & $\begin{array}{l}\text { 铁溶解 } \\
\text { Iron dissolution }\end{array}$ & $\begin{array}{l}\text { 碳分解 } \\
\text { Carbon decomposition }\end{array}$ & $\begin{array}{l}\text { 铁固定 } \\
\text { Iron sequestration }\end{array}$ & $\begin{array}{l}\text { 碳固定 } \\
\text { Carbon sequestration }\end{array}$ \\
\hline $\begin{array}{l}\text { 好氧 } \\
\text { Aerobic }\end{array}$ & $\begin{array}{l}\mathrm{a} \text { 光化学促进铁还原 } \\
\mathrm{b} \text { 有机配体促进铁矿物溶解 }\end{array}$ & $\begin{array}{l}\mathrm{a} \text { 微生物异养分解 } \\
\mathrm{b} \text { 活性氧驱动有机质氧化 }\end{array}$ & $\begin{array}{l}\mathrm{a} \text { 光合型亚铁氧化 } \\
\mathrm{b} \text { 好氧化学氧化 }\end{array}$ & $\begin{array}{l}\text { 光合型亚铁氧化耦合 } \\
\text { 碳同化 }\end{array}$ \\
\hline $\begin{array}{l}\text { 微氧 } \\
\text { Microaerobic }\end{array}$ & 有机配体促进铁矿物溶解 & 微生物异养分解 & 微氧型亚铁氧化 & $\begin{array}{l}\text { 微氧型亚铁氧化耦合 } \\
\text { 碳同化 }\end{array}$ \\
\hline $\begin{array}{l}\text { 厌氧 } \\
\text { Anaerobic }\end{array}$ & 微生物异养分解耦合铁还原 & $\begin{array}{l}\text { 微生物异养分解耦合 } \\
\text { 铁还原 }\end{array}$ & 自养型亚铁氧化 & $\begin{array}{l}\text { 自养型亚铁氧化耦合 } \\
\text { 碳同化 }\end{array}$ \\
\hline
\end{tabular}

\section{2 物理作用}

\subsection{1 铁矿物结合态有机碳的形成和分解}

有氧条件下铁矿物形态转化会影响土壤有机碳固定,归因于 $\mathrm{Fe}(\mathrm{II})$ 氧化转变成的铁矿物,会与土壤有机 碳结合形成稳定的铁矿物-有机碳复合物, 抵抗微生物分解, 从而增加长期土壤有机碳固定 ${ }^{[14,76-77]}$ 。另一方 面, $\mathrm{Fe}$ ( II) 离子浓度的下降导致酚氧化酶活性的降低并促进了土壤中水溶性芳香族化合物的积累,增强铁-木 质素结合, 以及抑制了水解酶的活性, 最终减少土壤有机碳的降解 ${ }^{[78]}$ 。然而, 最近研究开始逐渐关注厌氧条 件下异化铁还原这一过程。在戻氧条件下可溶性有机质通常分解不完全, 导致可溶性物质的累积,尤其是短 链脂肪酸 ${ }^{[79]}$, 而异化铁还原过程能耦合短链脂肪酸的氧化 ${ }^{[80-81]}$, 从而可能降低土壤有机碳固定。此外, 铁矿 物还原也会潜在的释放结合在铁矿物上的有机碳,从而影响铁矿物结合态有机碳的稳定性。最近一些关于氧 化还原反应过程中铁矿物结合态有机碳命运的研究,证实了铁还原反应的确会破坏铁矿物在稳定有机碳中的

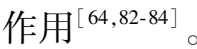

\subsection{2 团聚体的形成和解离}

铁氧化还原会通过影响土壤团聚体周转而改变土壤有机碳固定。铁矿物选择性地吸附芳香族有机化合 物形成铁矿物-芳香碳复合物,其会在黏粒和有机分子之间充当凝胶沉淀在黏粒表面,促进土壤团聚体的形 成。而当淹水时环境条件从有氧向厌氧转变时, 铁氧化物发生还原反应生成 $\mathrm{Fe}$ ( II), 其胶结作用减弱, 土壤 团聚体解离,会暴露更多新鲜表面以形成铁矿物-芳香碳复合物,随后在无氧向有氧条件转变过程中又会被重 新团聚所保护 ${ }^{[85]}$ 。氧化还原过程中产生的不同形态铁矿物对团聚体的影响程度不同。周而复始的氧化还原 更替,会促进短程有序的铁矿物产生 ${ }^{[86-87]}$, 其具有更大的表面积和高反应活性,对有机碳的稳定作用更强。 此外,也有研究表明,相对于游离态铁,无定型铁氧化物具有更强的胶结能力,对大团聚体的形成和稳定作用 可能更大 ${ }^{[88-90]}$ 。Jozefaciuk 和 Czachor ${ }^{[91]}$ 的研究则表明在自然状态下高含量的游离态铁会使团聚体稳定性更 强。Xue ${ }^{[92]}$ 等人发现在 $>0.25 \mathrm{~mm}$ 的团聚体中, 无定型态铁氧化物是最重要的粘结剂, 而在 $0.25-0.053 \mathrm{~mm}$ 团聚体中,无定型和络合态铁氧化物是主要的粘结剂。

\section{4 铁矿物形态转化影响有机碳固定的因素}

\section{1 气候}

当前研究认为铁对土壤有机碳的作用主要受温度、降雨调节。Doetterl ${ }^{[50]}$ 等横跨 $4000 \mathrm{~km}$ 测量了 24 个天 
然草地和灌木林的土壤和气候变量, 结果表明湿润地区的生物化学风化速率较高, 土壤有机碳组分主要为矿 物组分, 因此,有机-矿物相互作用形成的物理化学屏障阻碍了微生物利用土壤有机碳, 导致潮湿地区具有更 高的土壤有机碳储量; 而干旱地区的土壤风化主要受物理因素 (温度导致的机械粉碎) 驱动, 土壤有机碳矿物 组分较少,缺乏物理化学保护,使得分解者能轻易利用土壤有机碳,所以干旱地区 (具有极端高温和低温的温 度特征) 的土壤有机碳储量较低。然而, 也有研究认为寒冷干旱有利于铁/铝氧化物结合土壤有机碳 ${ }^{[21,93-94]}$ 。 Fang ${ }^{[95]}$ 等人对比不同气候区矿物质保护土壤碳的研究也发现寒冷干旱的西藏高寒草原的矿物质保护程度等 同于甚至更高于其他陆地生态系统, 如温带生态系统和热带-亚热带生态系统, 表明高山生态系统中土壤碳 固定对气候变暖的响应可能没有之前想象的那么敏感。

\section{2 土壤条件}

碱性阳离子 (例如 $\mathrm{K}^{+}, \mathrm{Mg}^{+}, \mathrm{Ca}^{2+}$ ) 的淋溶和铁、铝溶解度的增加可能通过改变吸附和共沉淀反应, 从而影 响矿物质结合态有机碳。研究表明土壤低 $\mathrm{pH}$ 下水解阳离子 (例如 $\mathrm{Fe}^{3+}$ 和 $\mathrm{Al}^{3+}$ ) 溶解度的增加, 会提高有机碳金属结合的稳定性 ${ }^{[96-97]}$ 。 $\mathrm{Ye}^{[98]}$ 等在内蒙古草地长期施氮和酸添加的试验中发现活性氮输人会显著的增加 根际外总土壤有机碳和轻组分碳含量, 而降低矿物质结合碳固定, 这主要归因于施氮导致的土壤酸化。一方 面其会降低微生物生长, 抑制微生物分解凋落物, 而微生物残体和木质素被认为是矿物质结合碳的重要来源 前体 ${ }^{[46,99-101]}$, 因此会降低矿物质结合态碳; 另一方面土壤酸化会降低铁铝矿物质与碳结合的 $\mathrm{pH}$ 敏感性, 提高 铁结合态有机碳, 降低钙结合态有机碳, 因此在矿物质结合态碳以钙/镁结合碳为主导的生态系统中, 长期施 氮导致的土壤酸化会降低土壤稳定碳的累积。

\section{3 铁矿物性质}

土壤有机碳与土壤矿物的结合通常归因于土壤矿物 (如层状硅酸盐, 称之为黏粒矿物) 具有较大的比表 面积, 会促进有机碳吸附到矿物基质 ${ }^{[60,102-103]}$ 。因此, 比表面积与土壤有机碳储量呈正相关关系, 以高比表面 积黏粒矿物为主导的土壤对腐殖质的吸附高于以低比表面积矿物质为主的土壤 ${ }^{[104]}$ 。铁 (氢) 氧化物具有高 的比表面积, 导致土壤有机碳和铁氧化物之间的正相关关系 ${ }^{[105-106]}$ 。低结晶度的水铁矿, 也因其具有高比表 面积 (最大可达 $800 \mathrm{~m}^{2} / \mathrm{g}$ ) ${ }^{[107-108]}$ 和表面丰富的羟基位点, 使其对有机大分子的吸附能力明显高于纤铁矿和针 铁矿 ${ }^{[109-111]}$ 。然而, 也有研究表明土壤比表面积与土壤有机碳没有很好的相关性, 比表面积和黏粒浓度最高 的土壤有机碳浓度并没有显著高于其他土壤 ${ }^{[112-113]}$ 。Hassink ${ }^{[114]}$ 也认为黏粒矿物的比表面积不是全球热带和 温带地区土壤固定有机碳潜力的良好指标。因此, 有关黏粒矿物表面积对土壤有机碳储量的重要性当前还没 有统一的认识。

铁矿物价态也可能影响其与有机碳的结合。为了验证不同价态铁矿物是否对可溶性有机质的分子分馏 作用不同, Wang ${ }^{[115]}$ 等选用四种具有代表性的铁矿物 (三价铁矿: 定型水铁矿和结晶度高的赤铁矿, 二价铁矿: 无定型硫化亚铁矿和结晶度高的黄铁矿) 与 DOM 进行吸附试验, 在好氧和厌氧条件下模拟 DOM 与铁矿物的 相互作用,并采用 ESI-FT-ICR MS 技术解析矿物对 DOM 的分子分馏机制。结果发现当以单位质量吸附剂 $(\mu \mathrm{g} \mathrm{C} / \mathrm{g})$ 为计量单位时, 铁矿物对 DOM 吸附容量的顺序为: 水铁矿>硫化亚铁矿>赤铁矿>黄铁矿; 但当以单 位比表面积吸附剂 $\left(\mu \mathrm{g} \mathrm{C} / \mathrm{m}^{2}\right)$ 为计量标准时, 吸附容量顺序为: 硫化亚铁矿>水铁矿>赤铁矿>黄铁矿。表明 水铁矿较高的吸附容量主要是由于其高比表面积和较强的静电作用, 而硫化亚铁是由于静电作用和同时具有 亲水性和疏水性的表面。水铁矿、赤铁矿和硫化亚铁矿均较强的吸附芳香碳,而黄铁矿的选择性吸附力较弱。 此外, 铁氧化物的价态也会直接影响共沉淀作用, 研究表明与土壤中可溶性有机碳发生共沉淀的为三价铁, 归 因于土壤有机碳对 $\mathrm{Fe}^{3+}$ 有很强的亲和力, 而对 $\mathrm{Fe}^{2+}$ 没有 ${ }^{[115]}$ 。当土壤中 $\mathrm{Fe}^{2+}$ 被氧化为 $\mathrm{Fe}^{3+}$, 然后发生水解反应 形成铁氧化物,其在有机碳存在的情况下同时形成就会发生共沉淀 ${ }^{[66,116]}$ 。

\section{4 碳源组成}

可溶性有机质中不同组分对矿物表面的亲和性不同 ${ }^{[96]}$, 被吸附到矿物表面的分子组分将被稳定保存,而 以较低亲和力官能团为特征的组分将留在水相中, 其更容易被微生物分解, 因此该选择性吸附机制为土壤有 
机碳的储存提供了思路 ${ }^{[15]}$ 。通常认为木质素的分子结构复杂, 具有一定化学抵抗性, 是减慢土壤有机碳分 解的重要贡献者 ${ }^{[37,117]}$ 。然而, 微生物效率-基质稳定性假说 (Mircobial Efficiency-Matrix Stabilization (MEMS) ) 认为可溶性物质来源的碳会有效地转化为微生物生物量和残体 (Necromass) (即高的碳利用效率的 化合物), 其易于与矿物质表面相互作用从而积累土壤有机质 ${ }^{[46,99,101,118]}$ 。相反的,复杂聚合物来源的碳例如 木质素, 有很低的微生物碳利用效率, 更可能以二氧化碳形式损失而不是贡献于矿物质结合态有机质 ${ }^{[101]}$ 。 然而, 也有研究认为这些没有充分考虑土壤有机质组分和矿物质之间化特异性相互作用的影响,其相互作用 在次生活性矿物比例较大的土壤中尤为重要 ${ }^{[119]}$ 。

最近研究认为铁氧化物会优先吸附或共沉淀木质素衍生的芳香碳和羧基碳 ${ }^{65,85,120-124]}$ 。Huang [100] 等人 通过添加 $\mathrm{C}_{4}$ 调落物和 $\mathrm{C}^{13}$ 标记木质素以追踪分解过程中木质素的命运, 结果发现只有 ( $\left.5.7 \pm 0.2\right) \%$ 木质素矿化 为 $\mathrm{CO}_{2}$, 而 $(48.2 \pm 0.9) \%$ 保存在矿物质结合态有机质中, 而且厌氧预处理时间越长木质素衍生的碳与铁结合 越多,表明微生物残体很重要,但木质素衍生的碳也可能对矿物质结合态有机质有不成比例的贡献,尤其是氧 化还原敏感的生物地球化学相互作用。进一步采用电喷雾-傅里叶变换-离子回旋共振质谱( ESI-FT-ICR-MS) 分析与水铁矿结合土壤有机碳的分子结构以及铁还原过程中释放的土壤有机碳的结构特征, 发现水铁矿优先 结合多环芳烃和维管植物来源的多酚, 而且在还原过程中这些组分不容易被释放。相比之下,脂肪碳则不易 被水铁矿结合,且在还原过程中,少量被水铁矿固定的脂肪碳也会被优先释放,表明了芳香性组分对矿物质结 合碳的稳定性具有重要作用 ${ }^{[64]}$ 。

\section{5 问题与研究展望}

当前有关土壤有机碳固持的地球化学机制已受到国内外多学科研究者的关注,现有研究表明铁氧化物会 促进土壤有机碳固定, 主要机制包括铁矿物通过吸附或共沉淀与土壤有机碳结合, 使其难以被微生物利用; 抑 制微生物生长和活性; 调节土壤酚氧化酶和水解酶活性等。当外界环境波动, 铁矿物形态转化则会通过多种 方式影响有机碳转化,如铁碳发生的生物-化学反应、影响铁矿物结合态有机碳形成和分解、影响团聚体的形 成和解离。这些过程会受到各种环境条件、自身的铁矿物性质和碳源质量等因素调控。尽管这些机制的研究 已经取得了一定的进展,但仍然存在很大的研究空间,建议今后多加强以下几方面的研究:

(1) 目前关于铁影响土壤有机碳固定的研究,多集中在有氧条件下,而对厌氧条件下关注较少。少量的 厌氧研究也只是关注异化铁还原过程, 对于厌氧条件下其他反应过程,如亚铁氧化,对土壤有机碳固定的作用 程度和方向的研究较缺乏, 建议今后的研究能更加全面系统的考虑铁循环的各反应过程。

(2) 目前有关铁氧化还原过程对有机碳累积的作用研究,多是对最终作用结果的简单定性研究, 而铁的 氧化和还原过程往往是同时发生, 对于中间过程还是 “黑箱”, 故建议加强氧化还原活跃土壤系统的研究, 以 及加人反应动力学研究和定量评估研究,阐明各反应过程对土壤有机碳固定的贡献。

(3) 当前关于土壤有机碳固持的地球化学机制研究多是基于无干扰或者是最小干扰的生态系统中。然 而, 自然环境下土壤有机碳累积是多因素相互作用的综合结果,各生态系统往往会受到不同形式和程度的人 为和自然干扰, 例如农田翻耕、施肥、草地放牧、森林火烧、湿地水位下降、水体富营养化、植物多样性降低、土 壤酸化等等问题,这些都会调控铁对土壤有机碳的固定作用。建议今后研究更多的关注干扰条件下铁循环对 土壤有机碳固定的作用和机理,可以更好地将理论模型与实际生态过程进行有机结合。

\section{参考文献 (References) :}

[ 1 ] Ilbert M, Bonnefoy V. Insight into the evolution of the iron oxidation pathways. Biochimica et Biophysica Acta (BBA)-Bioenergetics, 2013, 1827 (2) : 161-175.

[2] Karimian N, Johnston S G, Burton E D. Iron and sulfur cycling in acid sulfate soil wetlands under dynamic redox conditions: a review. Chemosphere, 2018, 197: 803-816.

[ 3 ] Tagliabue A, Bowie A R, Boyd P W, Buck K N, Johnson K S, Saito M A. The integral role of iron in ocean biogeochemistry. Nature, 2017, 543 
(7643) : 51-59.

[ 4 ] Keiluweit M, Bougoure J J, Nico P S, Pett-Ridge J, Weber P K, Kleber M. Mineral protection of soil carbon counteracted by root exudates. Nature Climate Change, 2015, 5(6): 588-595.

[ 5 ] Lalonde K, Mucci A, Ouellet A, Gélinas Y. Preservation of organic matter in sediments promoted by iron. Nature, 2012, 483(7388) : 198-200.

[ 6 ] Khomo L, Trumbore S, Bern C R, Chadwick O A. Timescales of carbon turnover in soils with mixed crystalline mineralogies. Soil, 2017, 3(1): 17-30.

[ 7 ] Schrumpf M, Kaiser K, Guggenberger G, Persson T, Kögel-Knabner I, Schulze E D. Storage and stability of organic carbon in soils as related to depth, occlusion within aggregates, and attachment to minerals. Biogeosciences, 2013, 10(3): 1675-1691.

[ 8 ] Jones E, Singh B. Organo-mineral interactions in contrasting soils under natural vegetation. Frontiers in Environmental Science, $2014,2: 2$.

[ 9 ] Han L F, Sun K, Jin J, Xing B S. Some concepts of soil organic carbon characteristics and mineral interaction from a review of literature. Soil Biology and Biochemistry, 2016, 94: 107-121.

[10] Lovley D R. Dissimilatory Fe( III) and Mn(IV) reduction. Microbiology and Molecular Biology Reviews, 1991, 55(2) : $259-287$.

[11] Hedrich S, Schlömann M, Johnson D B. The iron-oxidizing proteobacteria. Microbiology, 2011, 157(6) : 1551-1564.

[12] Picardal F. Abiotic and microbial interactions during anaerobic transformations of $\mathrm{Fe}$ ( II) and $\mathrm{NO}_{x}^{-}$. Frontiers in Microbiology, $2012,3: 112$.

[13] Roden E E, McBeth J M, Blothe M, Percak-Dennett E M, Fleming E J, Holyoke R R, Luther III G W, Emerson D, Schieber J. The microbial ferrous wheel in a neutral pH groundwater seep. Frontiers in Microbiology, 2012, 3: 172.

[14] Melton E D, Swanner E D, Behrens S, Schmidt C, Kappler A. The interplay of microbially mediated and abiotic reactions in the biogeochemical Fe cycle. Nature Reviews Microbiology, 2014, 12(12) : 797-808.

[15] Kappler A, Straub K L. Geomicrobiological cycling of iron. Reviews in Mineralogy and Geochemistry, 2005, 59(1) : 85- 108.

[16] Kampschreur M J, Kleerebezem R, de Vet W W J M, van Loosdrecht M C M. Reduced iron induced nitric oxide and nitrous oxide emission. Water Research, 2011, 45(18) : 5945-5952.

[17] Liu T X, Chen D D, Luo X B, Li X M, Li F B. Microbially mediated nitrate-reducing Fe( II) oxidation: quantification of chemodenitrification and biological reactions. Geochimica et Cosmochimica Acta, 2019, 256: 97-115.

[18] Lan Q, Li F B, Liu C S, Li X Z. Heterogeneous photodegradation of pentachlorophenol with maghemite and oxalate under UV illumination. Environmental Science \& Technology, 2008, 42(21) : 7918-7923.

[19] Bonneville S, Van Cappellen P, Behrends T. Microbial reduction of iron( III) oxyhydroxides: effects of mineral solubility and availability. Chemical Geology, 2004, 212(3/4): 255-268.

[20] Gault A G, Ibrahim A, Langley S, Renaud R, Takahashi Y, Boothman C, Lloyd J R, Clark I D, Ferris F G, Fortin D. Microbial and geochemical features suggest iron redox cycling within bacteriogenic iron oxide-rich sediments. Chemical Geology, 2011, 281(1/2) : 41-51.

[21] Adhikari D, Poulson S R, Sumaila S, Dynes J J, McBeth J M, Yang Y. Asynchronous reductive release of iron and organic carbon from hematitehumic acid complexes. Chemical Geology, 2016, 430: 13-20.

[22] 于天仁, 王振权. 土壤分析化学. 北京: 科学出版社, 1988.

[23] 陈家坊, 何群, 邵宗臣. 土壤中氧化铁的活化过程的探讨. 土壤学报, 1983, 20(4): 387-393.

[24] 何群, 陈家坊. 土壤中游离铁和络合态铁的测定. 土壤, 1983, 15(6): 242-244.

[25] 许祖诒, 陈家坊. 土壤中无定形氧化铁的测定. 土壤通报, 1980, 6: 32-35.

[26] 迟光宇, 张兆伟, 陈欣, 史奕. 差胺浸提-可见分光光度法测定土壤无定形铁. 光谱学与光谱分析, 2008, 28(12) : 2931- 2934.

[27] McKeague J A, Day J H. Dithionite- and oxalate-extractable Fe and Al as aids in differentiating various classes of soils. Canadian Journal of Soil Science, 1966, 46(1): 13-22.

[28] Byrne J M, Klueglein N, Pearce C, Rosso K M, Appel E, Kappler A. Redox cycling of Fe( II) and Fe( III) in magnetite by Fe-metabolizing bacteria. Science, 2015, 347(6229) : 1473-1476.

[29] Colombo C, Palumbo G, He J Z, Pinton R, Cesco S. Review on iron availability in soil: interaction of Fe minerals, plants, and microbes. Journal of Soils and Sediments, 2014, 14(3): 538-548.

[30] Li H, Hu S J, Polizzotto M L, Chang X L, Shen Q R, Ran W, Yu G H. Fungal biomineralization of montmorillonite and goethite to short-rangeordered minerals. Geochimica et Cosmochimica Acta, 2016, 191: 17-31.

[31] Sparks D L. Environmental Soil Chemistry. 2nd ed. Amsterdam: Academic Press, 2003.

[32] Smith P, Fang C M, Dawson J J C, Moncrieff J B. Impact of global warming on soil organic carbon. Advances in Agronomy, 2008, 97 : 1-43.

[33] Carvalhais N, Forkel M, Khomik M, Bellarby J, Jung M, Migliavacca M, Mu M Q, Saatchi S, Santoro M, Thurner M, Weber U, Ahrens B, Beer C, Cescatti A, Randerson J T, Reichstein M. Global covariation of carbon turnover times with climate in terrestrial ecosystems. Nature, 2014, 514 (7521) : 213-217. 
[34] Shao P, Zeng X B, Moore D J P, Zeng X D. Soil microbial respiration from observations and Earth System Models. Environmental Research Letters, 2013, 8(3): 034034 .

[35] Reeves D W. The role of soil organic matter in maintaining soil quality in continuous cropping systems. Soil and Tillage Research, 1997, 43( 1/2) : $131-167$.

[36] Lal R. Soil carbon sequestration impacts on global climate change and food security. Science, 2004, 304 (5677) : $1623-1627$.

[37] Schmidt M W I, Torn M S, Abiven S, Dittmar T, Guggenberger G, Janssens I A, Kleber M, Kögel-Knabner I, Lehmann J, Manning D A C, Nannipieri P, Rasse D P, Weiner S, Trumbore S E. Persistence of soil organic matter as an ecosystem property. Nature, 2011, 478(7367): 49-56.

[38] Yan J F, Wang L, Hu Y, Tsang Y F, Zhang Y N, Wu J H, Fu X H, Sun Y. Plant litter composition selects different soil microbial structures and in turn drives different litter decomposition pattern and soil carbon sequestration capability. Geoderma, 2018, 319: $194-203$.

[39] Lichtfouse É, Chenu C, Baudin F, Leblond C, Da Silva M, Behar F, Derenne S, Largeau C, Wehrung P, Albrecht P. A novel pathway of soil organic matter formation by selective preservation of resistant straight-chain biopolymers: chemical and isotope evidence. Organic Geochemistry, $1998,28(6): 411-415$.

[40] Lorenz K, Lal R, Preston C M, Nierop K G J. Strengthening the soil organic carbon pool by increasing contributions from recalcitrant aliphatic bio (macro) molecules. Geoderma, 2007, 142(1/2): 1- 10 .

[41] Wang X J, Butterly C R, Baldock J A, Tang C X. Long-term stabilization of crop residues and soil organic carbon affected by residue quality and initial soil pH. Science of the Total Environment, 2017, 587-588: 502-509.

[42] Xu Y D, Ding F, Gao X D, Wang Y, Li M, Wang J K. Mineralization of plant residues and native soil carbon as affected by soil fertility and residue type. Journal of Soils and Sediments, 2019, 19(3): 1407-1415.

[43] Lützow M V, Kögel-Knabner I, Ekschmitt K, Matzner E, Guggenberger G, Marschner B, Flessa H. Stabilization of organic matter in temperate soils: mechanisms and their relevance under different soil conditions-a review. European Journal of Soil Science, 2006, 57(4) : 426-445.

[44] Liang C, Schimel J P, Jastrow J D. The importance of anabolism in microbial control over soil carbon storage. Nature Microbiology, 2017, 2 (8) : 17105

[45] Miltner A, Bombach P, Schmidt-Brücken B, Kästner M. SOM genesis: microbial biomass as a significant source. Biogeochemistry, 2012, 111(1/ $3)$ : 41-55.

[46] Ma T, Zhu S S, Wang Z H, Chen D M, Dai G H, Feng B W, Su X Y, Hu H F, Li K H, Han W X, Liang C, Bai Y F, Feng X J. Divergent accumulation of microbial necromass and plant lignin components in grassland soils. Nature Communications, 2018, 9(1): 3480.

[47] Davidson E A, Janssens I A. Temperature sensitivity of soil carbon decomposition and feedbacks to climate change. Nature, 2006, 440 (7081) : $165-173$.

[48] Nie M, Pendall E, Bell C, Gasch C K, Raut S, Tamang S, Wallenstein M D. Positive climate feedbacks of soil microbial communities in a semiarid grassland. Ecology Letters, 2013, 16(2) : 234-241.

[49] Karhu K, Auffret M D, Dungait J A, Hopkins D W, Prosser J I, Singh B K, Subke J A, Wookey P A, Ågren G I, Sebastià M T, Gouriveau F, Bergkvist G, Meir P, Nottingham A T, Salinas N, Hartley I P. Temperature sensitivity of soil respiration rates enhanced by microbial community response. Nature, 2014, 513(7516): 81-84.

[50] Doetterl S, Stevens A, Six J, Merckx R, Van Oost K, Pinto M C, Casanova-Katny A, Muñoz C, Boudin M, Venegas E Z, Boeckx P. Soil carbon storage controlled by interactions between geochemistry and climate. Nature Geoscience, 2015, 8( 10): 780-783.

[51] Feng X J, Simpson A J, Simpson M J. Chemical and mineralogical controls on humic acid sorption to clay mineral surfaces. Organic Geochemistry, $2005,36(11): 1553-1566$.

[52] Kaiser K, Mikutta R, Guggenberger G. Increased stability of organic matter sorbed to ferrihydrite and goethite on aging. Soil Science Society of America Journal, 2007, 71(3): 711-719.

[53] Kleber M, Sollins P, Sutton R. A conceptual model of organo-mineral interactions in soils: self-assembly of organic molecular fragments into zonal structures on mineral surfaces. Biogeochemistry, 2007, 85(1): 9-24.

[54] Derenne S, Largeau C. A review of some important families of refractory macromolecules: composition, origin, and fate in soils and sediments. Soil Science, 2001, 166(11): 833-847.

[55] Knicker H. How does fire affect the nature and stability of soil organic nitrogen and carbon? A review. Biogeochemistry, 2007,85 (1): 91-118.

[56] Kögel-Knabner I, Guggenberger G, Kleber M, Kandeler E, Kalbitz K, Scheu S, Eusterhues K, Leinweber P. Organo-mineral associations in temperate soils: integrating biology, mineralogy, and organic matter chemistry. Journal of Plant Nutrition and Soil Science, 2008, 171( 1 ) : 61-82.

[57] Manjaiah K M, Kumar S, Sachdev M S, Sachdev P, Datta S C. Study of clay-organic complexes. Current Science, 2010, 98(7): 915-921.

[58] Schwarzenbach R P, Gschwend P M, Imboden D M. Environmental Organic Chemistry. Hoboken, NJ: Wiley-Interscience, 2003. 
[59] Six J, Conant R T, Paul E A, Paustian K. Stabilization mechanisms of soil organic matter: implications for C-saturation of soils. Plant and Soil, $2002,241(2): 155-176$

[60] Wagai R, Mayer L M. Sorptive stabilization of organic matter in soils by hydrous iron oxides. Geochimica et Cosmochimica Acta, 2007, 71 ( 1) : 2535 .

[61] Kaiser K, Guggenberger G. The role of DOM sorption to mineral surfaces in the preservation of organic matter in soils. Organic Geochemistry, 2000, $31(7 / 8): 711-725$.

[62] Eusterhues K, Wagner F E, Hausler W, Hanzlik M, Knicker H, Totsche K U, Kögel-Knabner I, Schwertmann U. Characterization of ferrihydritesoil organic matter coprecipitates by X-ray diffraction and Mossbauer spectroscopy. Environmental Science \& Technology, 2008 , 42 ( 21 ) : $7891-7897$.

[63] Mikutta R, Zang U, Chorover J, Haumaier L, Kalbitz K. Stabilization of extracellular polymeric substances (Bacillus subtilis) by adsorption to and coprecipitation with Al forms. Geochimica et Cosmochimica Acta, 2011, 75(11): 3135-3154.

[64] Han L F, Sun K, Keiluweit M, Yang Y, Yang Y, Jin J, Sun H R, Wu F C, Xing B S. Mobilization of ferrihydrite-associated organic carbon during Fe reduction: adsorption versus coprecipitation. Chemical Geology, 2019, 503: 61-68.

[65] Chen C M, Dynes J J, Wang J, Sparks D L. Properties of Fe-organic matter associations via coprecipitation versus adsorption. Environmental Science \& Technology, 2014, 48(23) : 13751-13759.

[66] Borggaard O K. Iron oxides in relation to aggregation of soil particles. Acta Agriculturae Scandinavica, 1983, 33( 3 ) : 257-260.

[67] Krull E S, Baldock J A, Skjemstad J O. Importance of mechanisms and processes of the stabilisation of soil organic matter for modelling carbon turnover. Functional Plant Biology, 2003, 30(2) : 207-222.

[68] Baldock J A, Skjemstad J O. Role of the soil matrix and minerals in protecting natural organic materials against biological attack. Organic Geochemistry, 2000, 31(7/8): 697-710.

[69] Sollins P, Swanston C, Kleber M, Filley T, Kramer M, Crow S, Caldwell B A, Lajtha K, Bowden R. Organic C and N stabilization in a forest soil: evidence from sequential density fractionation. Soil Biology and Biochemistry, 2006, 38(11) : 3313-3324.

[70] Amézketa E. Soil aggregate stability : a review. Journal of Sustainable Agriculture, 1999, 14(2/3): 83-151.

[71] Filimonova S, Kaufhold S, Wagner F E, Häusler W, Kögel-Knabner I. The role of allophane nano-structure and Fe oxide speciation for hosting soil organic matter in an allophanic Andosol. Geochimica et Cosmochimica Acta, 2016, 180: 284-302.

[72] Weiss J V, Emerson D, Megonigal J P. Rhizosphere iron ( III) deposition and reduction in a Juncus effusus L. -dominated wetland. Soil Science Society of America Journal, 2005, 69(6): 1861-1870.

[73] Widdel F, Schnell S, Heising S, Ehrenreich A, Assmus B, Schink B. Ferrous iron oxidation by anoxygenic phototrophic bacteria. Nature, 1993, $362(6423): 834-836$.

[74] Hall S J, Silver W L. Iron oxidation stimulates organic matter decomposition in humid tropical forest soils. Global Change Biology, 2013, 19(9) : 2804-2813.

[75] Mikutta R, Kleber M, Jahn R. Poorly crystalline minerals protect organic carbon in clay subfractions from acid subsoil horizons. Geoderma, 2005, $128(1 / 2): 106-115$.

[76] Rasmussen C, Southard R J, Horwath W R. Mineral control of organic carbon mineralization in a range of temperate conifer forest soils. Global Change Biology, 2006, 12(5): 834-847.

[77] Saidy A R, Smernik R J, Baldock J A, Kaiser K, Sanderman J, Macdonald L M. Effects of clay mineralogy and hydrous iron oxides on labile organic carbon stabilisation. Geoderma, 2012, 173-174: 104-110.

[78] Wang Y Y, Wang H, He J S, Feng X J. Iron-mediated soil carbon response to water-table decline in an alpine wetland. Nature Communications, $2017,8: 15972$.

[79] 吴金水, 葛体达, 胡亚军. 稻田土壤关键元素的生物地球化学耦合过程及其微生物调控机制. 生态学报, 2015, 35(20): 6626-6634.

[80] Hori T, Müller A, Igarashi Y, Conrad R, Friedrich M W. Identification of iron-reducing microorganisms in anoxic rice paddy soil by ${ }^{13}$ C-acetate probing. The ISME Journal, 2010, 4(2): 267-278.

[81] Ding L J, Su J Q, Xu H J, Jia Z J, Zhu Y G. Long-term nitrogen fertilization of paddy soil shifts iron-reducing microbial community revealed by RNA- ${ }^{13}$ C-acetate probing coupled with pyrosequencing. The ISME Journal, 2015, 9(3) : 721-734.

[82] Pan W N, Kan J J, Inamdar S, Chen C M, Sparks D. Dissimilatory microbial iron reduction release DOC ( dissolved organic carbon) from carbonferrihydrite association. Soil Biology and Biochemistry, 2016, 103: 232-240.

[83] Adhikari D, Zhao Q, Das K, Mejia J, Huang R X, Wang X L, Poulson S R, Tang Y Z, Roden E E, Yang Y. Dynamics of ferrihydrite-bound organic carbon during microbial Fe reduction. Geochimica et Cosmochimica Acta, 2017, 212: 221-233.

[84] Zhao Q, Adhikari D, Huang R X, Patel A, Wang X L, Tang Y Z, Obrist D, Roden E E, Yang Y. Coupled dynamics of iron and iron-bound 
organic carbon in forest soils during anaerobic reduction. Chemical Geology, 2017, 464 : 118-126.

[85] Huang X L, Tang H Y, Kang W J, Yu G H, Ran W, Hong J P, Shen Q R. Redox interface-associated organo-mineral interactions: a mechanism for $\mathrm{C}$ sequestration under a rice-wheat cropping system. Soil Biology and Biochemistry, 2018, 120: 12-23.

[86] Ginn B, Meile C, Wilmoth J, Tang Y Z, Thompson A. Rapid iron reduction rates are stimulated by high-amplitude redox fluctuations in a tropical forest soil. Environmental Science \& Technology, 2017, 51(6) : 3250-3259.

[87] Huang X L, Jiang H, Li Y, Ma Y C, Tang H Y, Ran W, Shen Q R. The role of poorly crystalline iron oxides in the stability of soil aggregateassociated organic carbon in a rice-wheat cropping system. Geoderma, 2016, 279: 1-10.

[88] Hou T, Xu R K, Tiwari D, Zhao A Z. Interaction between electrical double layers of soil colloids and Fe/Al oxides in suspensions. Journal of Colloid and Interface Science, 2007, 310(2) : 670-674.

[89] 郡宗臣，陈家坊. 几种氧化铁的离子吸附特性研究. 土壤学报，1984，21(2)：153-162.

[90] 王小红, 杨智杰, 刘小飞, 林伟盛, 杨玉盛, 刘志江, 赵本嘉, 苏瑞兰. 中亚热带山区土壤不同形态铁铝氧化物对团聚体稳定性的影响. 生态学报, 2016, 36(9): 2588-2596.

[91] Jozefaciuk G, Czachor H. Impact of organic matter, iron oxides, alumina, silica and drying on mechanical and water stability of artificial soil aggregates. Assessment of new method to study water stability. Geoderma, 2014, 221-222: 1- 10.

[92] Xue B, Huang L, Huang Y N, Yin Z Y, Li X K, Lu J W. Effects of organic carbon and iron oxides on soil aggregate stability under different tillage systems in a rice-rape cropping system. Catena, 2019, 177: 1-12.

[93] Mu C C, Zhang T J, Zhao Q, Guo H, Zhong W, Su H, Wu Q B. Soil organic carbon stabilization by iron in permafrost regions of the Qinghai-Tibet Plateau. Geophysical Research Letters, 2016, 43(19): 10286-10294.

[94] Zhao Q, Poulson S R, Obrist D, Sumaila S, Dynes J J, McBeth J M, Yang Y. Iron-bound organic carbon in forest soils: quantification and characterization. Biogeosciences, 2016, 13(16): 4777-4788.

[95] Fang K, Qin S Q, Chen L Y, Zhang Q W, Yang Y H. Al/Fe mineral controls on soil organic carbon stock across Tibetan alpine grasslands. Journal of Geophysical Research: Biogeosciences, 2019, 124(2) : 247-259.

[96] Gu B H, Schmitt J, Chen Z H, Liang L Y, McCarthy J F. Adsorption and desorption of natural organic matter on iron oxide: mechanisms and models. Environmental Science \& Technology, 1994, 28(1) : 38-46.

[97] Mueller K E, Eissenstat D M, Hobbie S E, Oleksyn J, Jagodzinski A M, Reich P B, Chadwick O A, Chorover J. Tree species effects on coupled cycles of carbon, nitrogen, and acidity in mineral soils at a common garden experiment. Biogeochemistry, 2012, 111( 1/3) : 601-614.

[98] Ye C L, Chen D M, Hall S J, Pan S, Yan X B, Bai T S, Guo H, Zhang Y, Bai Y F, Hu S J. Reconciling multiple impacts of nitrogen enrichment on soil carbon: plant, microbial and geochemical controls. Ecology Letters, 2018, 21(8) : 1162-1173.

[99] Lehmann J, Kleber M. The contentious nature of soil organic matter. Nature, 2015, 528(7580): 60-68.

[ 100] Huang W J, Hammel K E, Hao J L, Thompson A, Timokhin V I, Hall S J. Enrichment of lignin-derived carbon in mineral-associated soil organic matter. Environmental Science \& Technology, 2019, 53(13): 7522-7531.

[101] Cotrufo M F, Wallenstein M D, Boot C M, Denef K, Paul E. The Microbial Efficiency-Matrix Stabilization ( MEMS) framework integrates plant litter decomposition with soil organic matter stabilization: do labile plant inputs form stable soil organic matter? Global Change Biology, 2013 , 19 (4) : 988-995.

[102] Balabane M, Plante A F. Aggregation and carbon storage in silty soil using physical fractionation techniques. European Journal of Soil Science, $2004,55(2): 415-427$.

[103] Tipping E. The adsorption of aquatic humic substances by iron oxides. Geochimica et Cosmochimica Acta, 1981, 45(2) : $191-199$.

[104] Tate K R, Theng B K G. Organic matter and its interactions with inorganic soil constituents//Theng B K G, ed. Soils with Variable Charge. New Zealand: Society of Soil Science, 1980: 225-249.

[105] Kaiser K, Guggenberger G. Mineral surfaces and soil organic matter. European Journal of Soil Science, 2003, 54(2) : 219-236.

[106] Kleber M, Mikutta R, Torn M S, Jahn R. Poorly crystalline mineral phases protect organic matter in acid subsoil horizons. European Journal of Soil Science, 2005, 56(6): 717-725.

[ 107] Eusterhues K, Rumpel C, Kögel-Knabner I. Stabilization of soil organic matter isolated via oxidative degradation. Organic Geochemistry, 2005 , 36 (11) : 1567-1575.

[108] Goldberg S. Application of surface complexation models to anion adsorption by natural materials. Environmental Toxicology and Chemistry, 2014, $33(10): 2172-2180$.

[109] Kaiser K, Guggenberger G, Haumaier L, Zech W. Dissolved organic matter sorption on sub soils and minerals studied by ${ }^{13}$ C-NMR and DRIFT spectroscopy. European Journal of Soil Science, 1997, 48(2): 301-310.

[110] Chorover J, Amistadi M K. Reaction of forest floor organic matter at goethite, birnessite and smectite surfaces. Geochimica et Cosmochimica Acta, 
2001, 65(1): 95- 109 .

[111] Kleber M, Nico P S, Plante A, Filley T, Kramer M, Swanston C, Sollins P. Old and stable soil organic matter is not necessarily chemically recalcitrant: implications for modeling concepts and temperature sensitivity. Global Change Biology, 2011, 17(2) : 1097-1107.

[112] Mayer L M, Xing B S. Organic matter-surface area relationships in acid soils. Soil Science Society of America Journal, 2001, 65(1): $250-258$.

[113] Wiseman C L S, Puttmann W. Soil organic carbon and its sorptive preservation in central Germany. European Journal of Soil Science, 2005,56 (1): $65-76$.

[114] Hassink, J. The capacity of soils to preserve organic C and N by their association with clay and silt particles. Plant Soil, 1997, 191(1) : 77-87.

[115] Wang Y, Zhang Z Y, Han L F, Sun K, Jin J, Yang Y, Yang Y, Hao Z N, Liu J F, Xing B S. Preferential molecular fractionation of dissolved organic matter by iron minerals with different oxidation states. Chemical Geology, 2019, 520: 69-76.

[116] Mikutta R, Lorenz D, Guggenberger G, Haumaier L, Freund A. Properties and reactivity of Fe-organic matter associations formed by coprecipitation versus adsorption: clues from arsenate batch adsorption. Geochimica et Cosmochimica Acta, 2014, 144: 258- 276.

[117] Thevenot M, Dignac M F, Rumpel C. Fate of lignins in soils: a review. Soil Biology and Biochemistry, 2010, 42(8) : 1200-1211.

[118] Sokol N W, Bradford M A. Microbial formation of stable soil carbon is more efficient from belowground than aboveground input. Nature Geoscience, 2019, 12(1): 46-53.

[119] Sokol N W, Sanderman J, Bradford M A. Pathways of mineral-associated soil organic matter formation: integrating the role of plant carbon source, chemistry, and point of entry. Global Change Biology, 2019, 25(1): 12-24.

[120] Riedel T, Zak D, Biester H, Dittmar T. Iron traps terrestrially derived dissolved organic matter at redox interfaces. Proceedings of the National Academy of Sciences of the United States of America, 2013, 110(25) : 10101-10105.

[121] Eusterhues K, Rennert T, Knicker H, Kogel-Knabner I, Totsche K U, Schwertmann U. Fractionation of organic matter due to reaction with ferrihydrite: coprecipitation versus adsorption. Environmental Science \& Technology, 2010, 45(2): 527-533.

[ 122] Kramer M G, Sanderman J, Chadwick O A, Chorover J, Vitousek P M. Long-term carbon storage through retention of dissolved aromatic acids by reactive particles in soil. Global Change Biology, 2012, 18(8): 2594-2605.

[123] Hall S J, Silver W L, Timokhin V I, Hammel K E. Iron addition to soil specifically stabilized lignin. Soil Biology and Biochemistry, 2016, 98 : 95-98.

[124] Coward E K, Ohno T, Plante A F. Adsorption and molecular fractionation of dissolved organic matter on iron-bearing mineral matrices of varying crystallinity. Environmental Science \& Technology, 2018, 52(3) : 1036-1044. 